\title{
Antimicrobial properties and application of fig seed oil as an additive for chitosan-based films
}

\author{
Dilruba Baykara, Esra Pilavcı, \\ Mehdi Meran, Zihni Onur Çalışkaner
}

Üsküdar University, İstanbul, Turkey

Keywords:

Antimicrobial

Films

Packaging

Fig seed

Oil

\section{Article history:}

Received 29.08.2020

Received in revised form 18.02.2021

Accepted 30.06.2021

Corresponding author:

Zihni Onur Çalışkaner

E-mail:

zihnionur.caliskaner@ uskudar.edu.tr

DOI: $10.24263 / 2304-$

974X-2021-10-2-7

\section{Abstract}

Introduction. In this study, we aimed to test whether the antimicrobial capacity of chitosan polymer could be increased synergistically when fig seed oil, plum kernel oil, apricot kernel oil were applied in different conditions.

Materials and methods. Antimicrobial effects of these oils against to certain bacterial species were evaluated with/without chitosan by agar disk/well diffusion and spectrophotometric measurement. Thin chitosan films enriched by seed oils were produced to test both antifungal and antibacterial effects in vivo and also in application.

Results and discussion. Although we could not obtain significant effect in culture conditions, fig seed oil singly or in combination with apricot and plum kernel oil was able to improve anti-spoilage properties of chitosan film. While fresh lemon and banana slices wrapped only ordinary cling film showed a complete deterioration, chitosan-film could substantially prohibit microbial spoilage. Moreover, almost a complete protection against microbial spoilage has been determined for these food particularly packed by chitosanfilm enriched with fig seed oil. Besides, MD simulation was performed in order to evaluate putative interactions between oil compounds and chitosan. We have proposed that the most potential compound which is common in all theree oil extracts, is benzaldehyde. Herein, interaction through $\mathrm{H}$-bonding was determined between between functional groups of the chitosan and benzaldehyde molecule by computational analysis, suggesting this might be one of possible factors for the observed contribution of fig seed oil and also other oil extracts to anti-spoilage effects of chitosan-based packaging film.

Conclusions. Addition of fig seed oil alone or in combination with various extracts into food packages has an application potential in order to extend the shelf life of food by alleviating deterioration for several days. 


\section{- Food Technology -}

\section{Introduction}

Food deterioration or spoilage as a result of various chemical, biological, and/or physical changes, is a serious problem which is encountered during processes such as production, storage and distribution (Kong and Singh, 2011). Changes in mentioned features cause food to become unsuitable for consumption due to loss in tissue integrity, nutritional values, chemical/biological activity and benefit. Spoilage does not only render a food harmful and risky for human and public health but also causes economic losses (Rawat, 2015). The factors that cause food spoilage are classified in three main groups as chemical, physical and microbial (Odeyemi et al., 2020). Microorganisms such as bacteria and fungi (mold, yeast) are the leading biological agents which give rise to food spoilage. Some compounds produced by microorganisms on the food ruin the physical and biochemical properties such as colour, texture, nutrient content of the food and consequently poison the food (Rawat, 2015). Spoilage microorganisms include mold species such as Penicillium, Aspergillus, yeast species such as Saccharomyces, Candida and bacterial species such as Escherichia, Bacillus, Staphylococcus (Sperber, 2009). A variety of traditional and modern approaches such as drying, freezing, heat treatment and antimicrobial packaging, respectively, are usually applied to avoid or retard food spoilage (Amit et al., 2017).

Antimicrobial packaging, a relatively current approach, is based on the principle of constitution of a protective layer against to saprophyte microorganisms outside the food by incorporating antimicrobial agents in the packaging film (Malhotra et al., 2015). Certain natural essential oils from plants, as well as polymers such as polyvinylchloride (PVC), polypropylene (PP), high density polyethylene (HDPE), low density polyethylene (LDPE), polyethylene terephthalate (PET), polylactic acid (PLA) and chitosan are frequently prefered substances in antimicrobial films (Huang et al., 2019). The best example of antimicrobial polymers is chitosan with chemical formula [(C6H11NO4)n (C8H13NO4)m] (Marpu and Benton, 2018; Mahira et al., 2019). Chitosan has the opportunity to be used in many areas, especially food industry, and has superior properties compared to many other biopolymers in terms of its chemical and physical properties since it is prevalent and abundant in nature, less or non-toxic in human beings, biodegradable, biocompatible and antimicrobial (Marpu and Benton, 2018; Tan et al., 2015). Studies have shown that chitosan has inhibitory effects on the growth and reproduction of many microorganisms such as Escherichia coli, Staphylococcus sp., Bacillus sp., Salmonella sp., Listeria sp., Micrococcus sp., Vibrio sp., etc. (Goy et al., 2009). However, it has been determined that coating the food with chitosan film reduces the partial oxygen pressure in the package, contributes to keeping the temperature in balance by transfer of moisture between the food and its environment, prevents loss of water and delays enzymatic browning in fruits (Kahve and Duran, 2016).

On the other hand, essential and fixed oils, obtained from different parts of plants, such as flowers, buds, leaves, fruits, shells, seeds, have quite different biological activities and are another most widely used additives in the food industry (Huang et al., 2019). Some of the oils also show antimicrobial activity by altering membrane permeability of the microbial cells, causing cytotoxic effect or disrupting the cellular energy system (Chouhan et al., 2017). Due to such antimicrobial effects, certain oils are repurposed or tested in antimicrobial films/packages. For instance, grapefruit seed extract has been shown to exhibit a broad inhibition on microbial growth against both gram-positive and gram-negative bacteria (Heggers et al., 2004). In addition, chitosan-based composite films containing grapefruit seed extract as an antimicrobial and antifungal agent have been reported to have the potential to delay the incidence of fungal growth (Tan et al., 2015). In another study, various oils extracted from the seeds of jackfruit (Artocarpus heterophyllus), papaya (Carica papaya), 
banana peels (Musa acuminata), tamarind (Tamarindus indica), pineapple (Ananas comosus), plum (Prunus domestica), musambi (Citrus limetta), strawberry (Fragaria ananassa), orange (Citrus sinensis) and guava (Psidium guajava) were screened for their potential antimicrobial activities (Debnath et al., 2011). Although extracts of banana, pineapple and musambi did not show any significant microbial activity contrary to bacterial and fungal strains, plum seed oil has been showed to have the strongest broad spectrum antibacterial activity and antifungal activity (Debnath et al., 2011; Savic et al., 2016). It has also reported that essential oil from apricot kernel had antimicrobial activity against various bacteria and yeasts (Lee et al 2014) and combining chitosan and apricot kernel oil in the packaging film has been shown to increase the antimicrobial effect and contributed to food preservation (Priyadarshi et al., 2018).

Despite the fact that presence of certain studies on antimicrobial properties of apricot kernel oil and plum kernel oil and their cooperation in chitosan films, the number of researches dealing with fig seed oil is almost negligible. The only existing study which published by Duman et al., revealed the chemical content of fig seed oil and demonstrated that fig seed oil had a great antifungal effect against Candida albicans and Aspergillus flavus strains and strong antibacterial effect on Staphylococcus aureus, Pseudomonas aeruginosa, Escherichia coli, Enterococcus faecalis but also weak effect on P. aeruginosa and Klebsiella pneumoniae in vitro (Duman et al., 2018). Nevertheless, application of fig seed oil as an additive in chitosan-based antimicrobial films has not been reported yet.

As it was reviewed above, the use of antimicrobial polymers such as chitosan in food packages are crucial to prevent food spoilage in order to extend shelf life. However, there are several ongoing studies about investigation of additives that are easily accessible, biocompatible, biodegradable, non-hazardous for the health and have low cost but high antimicrobial potential for antimicrobial films.

From the view of this point, we aimed to evaluate antimicrobial effects of fig seed oil singly and in combination with apricot kernel oil and plum kernel oil synergistically both in vitro and in vivo.

For this purpose, we have performed antimicrobial tests such as agar disk diffusion, agar well diffusion and spectrophotometric measurement against to Escherichia coli and Bacillus subtilis in the culture. Along with this, thin chitosan-based films including each oil individually and simultaneously together, were produced and tested against both saprophyte bacteria and fungi on various food by observing microorganismal colonization and by tracking the changes in physical conditions such as texture, browning, odour, rigidity, etc. Herein we demonstrated that fig seed oil alone and combination of fig seed oil, apricot kernel oil and plum kernel oil in chitosan-film conserved the food for a longer time rather than the film including chitosan only and hence we concluded that fig seed oil and the others ultimately exhibited a synergistic anti-spoilage effect in practice. Thus it has been the first study that examined the usage potential of these oils synergistically proposing a promising usage potential of fig seed oil and also in cooperation with tested oils for the antimicrobial packaging applications in near future.

\section{Material and methods}

\section{Chitosan and seed oil extracts}

Chitosan (medium molecular weight) was obtained from Sigma Aldrich (\#448877-50G). The marketed seed oil extracts were supplied from reliable manufacturers in Turkey. Apricot 


\section{— Food Technology —}

seed oil (Prunus armeniaca) and fig seed oil (Ficus carica) were purchased from Arifoğlu Co. (İstanbul, Turkey). Plum seed oil (Prunus salicina) was obtained from Neva Co. (İstanbul, Turkey). LB Broth and LB Agar (Caisson Labs, UT, USA) were prepared according to manufacturer's instructions.

\section{Film preparation}

$2 \%(\mathrm{w} / \mathrm{v})$ of chitosan polymer was dissolved in $1 \%(\mathrm{v} / \mathrm{v})$ aqueous acetic acid (Merck, NJ, USA) solution. Solution was stirred at $70^{\circ} \mathrm{C}$ for 48 hours to obtain a completely homogeneous gel-like structure in liquid form. Tween 80 (0.2\%, v/v) (Merck, NJ, USA) as an emulsifier and each oil $(1.5 \%, \mathrm{v} / \mathrm{v})$ according to experimental design (Table 1) were added into solution. First experimental group was prepared as $100 \mathrm{ml}$ chitosan solution only. Each mixture was spread on the plates covered with cling film and waited for solidification at room temperature for 24 hours. By the way, one of the surface of a piece of ordinary cling film has covered with chitosan-based film as well.

\section{Antimicrobial activity}

Antimicrobial tests were carried out by using LB broth medium and LB agar-related methods. For agar well diffusion method Escherichia coli and Bacillus subtilis were streaked through the LB agar as widespread and then 1-mm diameter wells were punctured in agar to load $20 \mu \mathrm{l}$ of solution. Concentration for oils and chitosan were used equivalent to amount in the film. In disk diffusion method, round pieces with $1.5-\mathrm{mm}$ diameter were cut from the prepared thin films and placed on LB agar spreaded with bacteria. Petri dishes were incubated at $37^{\circ} \mathrm{C}$ overnight to measure inhibition zone around the wells or disks. Along with this, antimicrobial test were also performed by cultivation in LB broth liquid media. Chitosan and each oil were added into LB broth in a final concentration of $1.5 \%(\mathrm{w} / \mathrm{v})$ and $1.5 \%(\mathrm{v} / \mathrm{v})$, respectively. Upon incubation at $37^{\circ} \mathrm{C}$ for overnight, optic density (OD) was measured at 600 $\mathrm{nm}$ by UV spectrophotometer (Schimadzu, Japan) to compare relative bacterial growth between experimental groups.

\section{Calculation of \% Inhibition Rate}

Escherichia coli and Bacillus subtilis were cultured in LB broth media containing chitosan only, seed oil extracts only and both in combination (Table 1). Bacterial growth in such conditions was measured at $600 \mathrm{~nm}$ wavelength to evaluate inhibitory effect relative to control by calculating \% inhibition rate of substances alone or in combination. The following equation was used to calculate the percentage of inhibition on bacterial growth relative to control which does not contain either chitosan and oil in LB by using absorbance values (A) (Rolim et al., 2015).

$$
\% \text { inhibition }=\frac{(\text { Acontrol }- \text { Asample })}{\text { Acontrol }} \times 100
$$

Absorbance obtained for each sample (Asample) was subtracted from the absorbance of control (Acontrol). Lastly, \% inhibition rate was calculated by division of subtraction by the Acontrol and multiplication by \%. Hence inhibition in control was accounted as $0 \%$ and inhibition rate in experimental groups have been calculated relatively compared to control. Experiment was performed in triplicate for Escherichia coli and average results were exploited for further analysis while it's attempted for only once for Bacillus subtilis. 


\section{Simulation}

Molecular dynamics (MD) simulation was employed to explore the interaction between benzaldehyde and chitosan polymer chain. Then number of repeating unit for the polymer was considered to be 19 . The ratio of the polymer/benzaldehyde molecules was considered to be 1/10. Canonical ensemble, NVT (constant number of molecules, constant volume, and constant temperature), at $300 \mathrm{~K}$ were used to implement the molecular dynamics for a total duration of $5.0 \mathrm{~ns}$ using a $1.0 \mathrm{fs}$ time step. AMBER force field (Bayly et al., 1995) was also used in the MD. All the simulations were performed using the LAMMPS software package (Plimpton, 1995).

\section{Results and discussion}

\section{Antimicrobial test of chitosan, seed oil extracts and chitosan-seed oil combinations on agar culture}

It was determined that the inhibition zones around the wells were quite small and there was no significant difference between the control and other groups. Observed inhibition zones were less than expected and these zones were not sufficient to measure and compare accurately since they were about/under only $1 \mathrm{~mm}$ on the agar (data not shown). The results were similar for both types of bacteria. Because we have thought that oil extracts which were directly loaded in wells may not have been able to form an effective inhibition zone due to their inability to diffuse in water-based agar. We have attempted disk diffusion method to overcome this trouble. However, there was no notable and comparable inhibition zones around the films on agar plates seeded by Escherichia coli and Bacillus subtilis (data not shown). There were only restricted inhibition zones around contact areas of the well and film containing chitosan alone (data not shown).

Chitosan, apricot kernel oil, plum kernel oil, fig kernel oil, and chitosan-seed oil solutions were tested on Bacillus subtilis and Escherichia coli bacteria using both agar well diffusion and agar disk diffusion methods in obedience to Table 1.

Table 1

\section{Design of control and experimental groups}

\begin{tabular}{|l|c|c|c|c|}
\hline Film Type & Chitosan & Apricot Kernel Oil & Plum Kernel Oil & Fig Seed Oil \\
\hline Control & - & - & - & - \\
\hline Ch & + & - & - & - \\
\hline Ch+AKO & + & + & - & - \\
\hline Ch+PKO & + & - & + & - \\
\hline Ch+FSO & + & - & - & + \\
\hline Ch+Triple & + & + & + & + \\
\hline
\end{tabular}




\section{Antimicrobial test of chitosan, seed oil extracts and chitosan-seed oil combinations in broth medium}

Results showed that chitosan alone was the most effective reducing $E$. coli growth by $48 \%$, and unexpectedly the oil extracts could not contribute to antibacterial effect of chitosan in culture conditions (Table 2 and Figure 1).

Average absorbance values and calculated \% inhibition rates for Escherichia coli

Table 2 (average of 3 independent experiments)

\begin{tabular}{|l|c|c|}
\hline Film Type & Mean Absorbance & Calculated Inhibition Rate (\%) \\
\hline Control & 1.271 & 0 \\
\hline Ch+AKO & 1.105 & 13 \\
\hline Ch+PKO & 1.077 & 15 \\
\hline Ch+FSO & 0.937 & 26 \\
\hline Ch+Triple & 1.011 & 20 \\
\hline Ch & 0.650 & 48 \\
\hline
\end{tabular}

These unexpected results may have caused by the concentration of oils and density of bacterial culture. Similar with $E$. coli, chitosan was the most effective agent alone reducing bacterial growth by $92 \%$ (data not shown). While $\mathrm{Ch}+\mathrm{AKO}$ reduced growth by $53 \%$, $\mathrm{Ch}+\mathrm{PKO}, \mathrm{Ch}+$ Triple and $\mathrm{Ch}+\mathrm{FSO}$ had inhibition on bacterial growth with $46 \%, 45 \%$ and $25 \%$, respectively obtained from only single experimental attempt (data not shown). We preliminarily showed each oil with chitosan has relatively more bactericidal activity on $B$. subtilis, a gram-positive species. It was complied with a study reported gram-positive bacteria was more sensitive against certain essential oils rather than gram-negative bacteria (Erkmen \& Özcan 2004). That was the reason why preliminary inhibition rates was high in Bacillus subtilis.

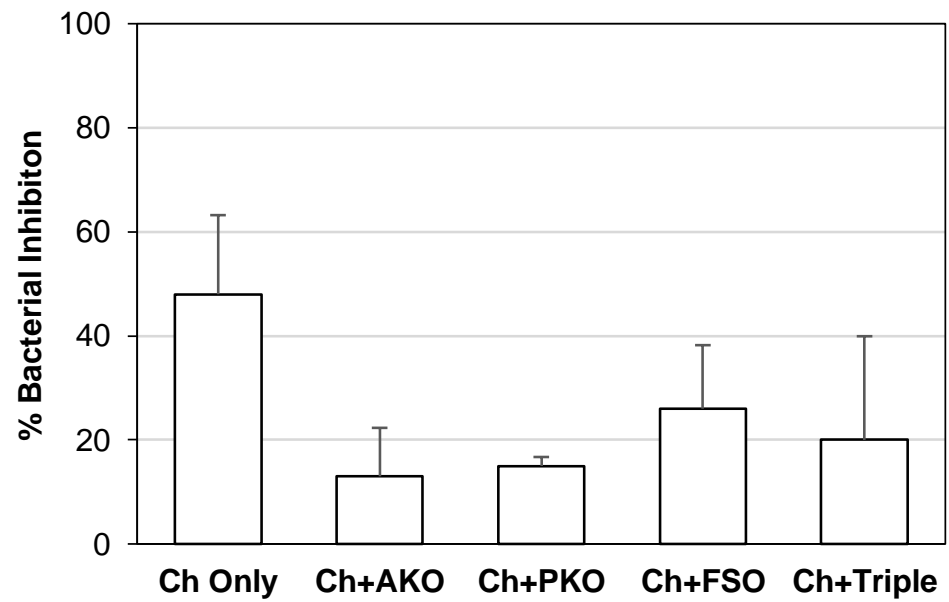

Figure 1. Relative inhibition rates on $E$. coli growth (Error bars represent SEM. \#ns = statictically non-significant) 


\section{Contribution of Seed Oil Extracts to Protective Effects of Antimicrobial Chitosan Films}

Chitosan-included films are already used in antimicrobial packaging. Even though we were not able to consistent results in culture conditions, we tested the usage and antimicrobial potential of apricot kernel oil, plum kernel oil and fig seed oil in the chitosan film-related application. For this purpose, we incorporated each oil extract alone or all of them synergistically into thin chitosan films and various fresh and well-conditioned foods (such as lemon, banana, bread and cheese) were packed. Slices with the similar size obtained from only the same food were preferred for packaging in order to eliminate variability and inequality such as genetic, morphological, biochemical and/or productional discrepancy. As a control, some pieces were wrapped by only cling film to isolate food from aerial environment. All of experimental groups and controls were kept in the same place and conditions (dry, dark, room temperature) and visible changes were regularly observed for 9 days.

We could acquire the most appreciable and dramatic results for lemon slices and afterward banana. At the end of the 9th day, control slice was completely decayed by observable microorganismal propagation and physical changes such as browning, dehydratation and so on (Figure 2 and Table 3). The slice wrapped by only chitosan film has stayed more durable than the control as expected even there was a trace of degradation. However, chitosan films with AKO, PKO and FSO, singly and in triplet could keep slices in more unspoilt condition relative to chitosan film alone. Along with this, same results were achieved for also banana slices. While control evolved into a bad manner, chitosan film could protect the fruit and the slice clinged with $\mathrm{Ch}+$ Triple film was the piece in the best condition (Table 3). However, we could not detect significant difference in the condition of bread and cheese among $\mathrm{Ch}$ film and $\mathrm{Ch}$ films including oil extracts (data not shown).

Table 3

Food spoilage rates detected according to sensory parameters such as color, odor, hardness, water loss, texture deterioration, and etc.

Deterioration level is relatively gradated between " $+++++"$ " = most deterioration) and "+" (= least deterioration)

\begin{tabular}{|l|c|c|}
\hline Film Type & Degradation Rate of Lemon Slice & Degradation Rate of Banana Slice \\
\hline Control & +++++ & ++++ \\
\hline Ch & +++ & +++ \\
\hline Ch+AKO & + & ++ \\
\hline Ch+PKO & ++ & ++ \\
\hline Ch+FSO & + & +++ \\
\hline Ch+Triple & + & + \\
\hline
\end{tabular}




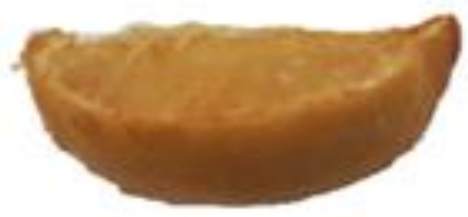

$\boldsymbol{a}$

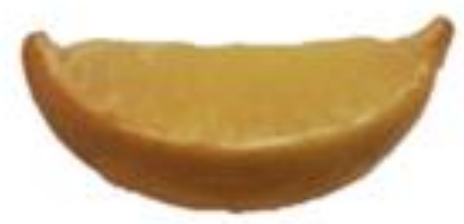

$c$

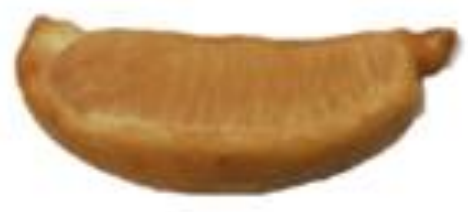

$\boldsymbol{e}$

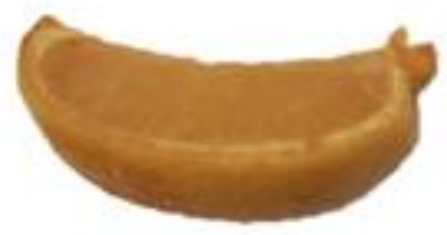

$\boldsymbol{b}$

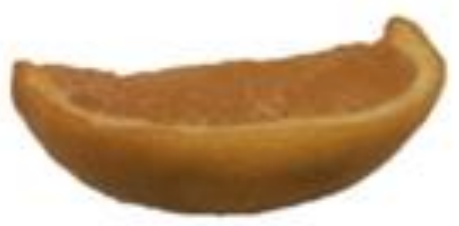

d

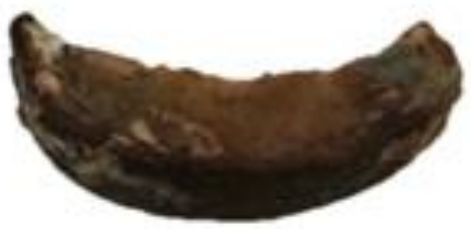

$f$

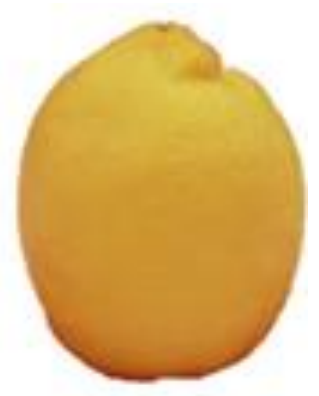

$\boldsymbol{g}$

Figure 2. Captured images of lemon samples covered by chitosan-based films 9 days after packaging.

a: $\mathrm{Ch}, \mathbf{b}: \mathrm{Ch}+\mathrm{FSO}$,

c: $\mathrm{Ch}+\mathrm{AKO}, \mathbf{d}: \mathrm{Ch}+\mathrm{PKO}$,

e: Ch+Triple, f: Control,

g: Status of the material in the beginning (Any post-image processing was not applied on the raw images except cropping) 


\section{Molecular Dynamics Simulation of Candidate Active Substance In Seed Oils and Chitosan}

Benzaldehyde is an aromatic aldehyde of plant metabolites as flavouring agent and one of the mostly found active organic compound in apricot, plum (Verma et al., 2017; Vyviurska et al., 2017) and also in fig (Russo et al., 2017; Mujić et al., 2014). It has also been reported that benzaldehyde is one of the constituent that gains antibacterial, antifungal and antioxidant properties to the certain essential oil extracts including apricot kernel oil (Eissen et al., 2015; Alamri et al., 2012; Geng et al., 2016). At this point we have speculated and thus examined computationally whether benzaldehyde, a common ingredient in tested oils, could contribute effects of chitosan by interacting in films anywise. In order to test this capability, we performed Molecular Dynamics (MD) simulation.

The configuration of the benzaldehyde molecule on the chitosan polymer chain is represented in Figure 3. It can be seen that hydrogen bonding build up between $-\mathrm{NH} 2$ functional groups of the chitosan and carbonyl group of the benzaldehyde molecule. It can be expected that the interaction of the benzaldehyde molecules with the polymer chain have the ability to increase the antimicrobial activity of the chitosan due to the introduction of hydrophobicity to the polymer chain (Goy et al 2009). However, chitosan is an amphiphilic polymer (Sahariah and Másson, 2017), certain studies demonstrated that the antibacterial activity of the chitosan had improved with an increasing on the chain length of the alkyl substituent (Rabea et al., 2003; Inta et al., 2014). In these studies, researchers found that the activity could be attributed to the contribution of the hydrophobic portions on the polymer. Tamer et al. has also reported that two derivatives of chitosan prepared by 4-chloro benzaldehyde and benzophenone structures had improved the antibacterial activity of the polymer against both Gram-positive and Gram-negative strains (Tamer et al., 2016). Additionally, it was shown that N-benzylidene chitosan, synthesized by chitosanbenzaldehyde interaction, had better adsorption properties to metals and higher chemical resistance than that of chitosan (Thien et al., 2017). N-acylated chitosan was also assessed to be favorable for the interaction of polymer molecule and bacterial cell as a result of enriched hydrophobicity in polymer (Kong et al., 2010; Hu et al., 2007). On the other hand, it was investigated that antimicrobial activity of chitosan in lipid emulsions was higher than in aqueous solutions (Jumaa et al., 2002). Hereby, we have proposed that benzaldehyde might be one of the compound in the fig and other fruits which improves the chemical properties of chitosan and that could possibly be one of the reasons of upgrading anti-spoilage properties of films enriched with fig oil alone and in combination but not a significant bactericidal effect in water-based culture conditions.

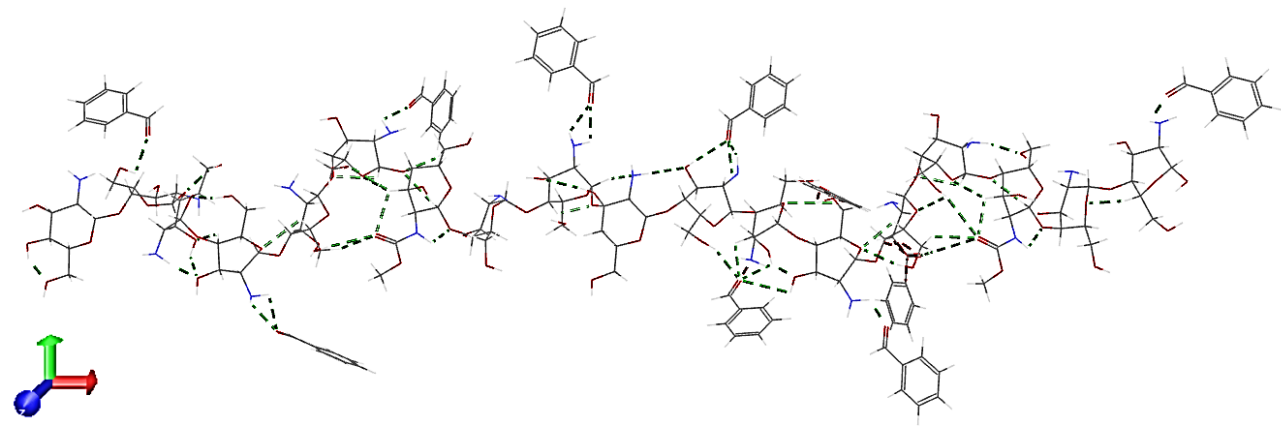

Figure 3. Hydrogen bond formation between benzaldehyde and-HN2 groups of chitosan 


\section{Conclusion}

Herein, apricot kernel oil, fig seed oil and plum kernel oil in order showed a fine antispoilage activity by inhibiting the growth of saprophyte microorganisms such as bacteria and mold on the food. Moreover cooperation of three oil extracts had a more and synergistic protective effect contrary to deterioration of both fruits. With this study, we have demonstrated the anti-spoilage effect and usage potential of fig seed oil singly and together with other tested oils in the chitosan-based films for antimicrobial packaging, for the first time. Hence, we have concluded that fig seed oil can contribute antimicrobial effect of chitosan by alone and/or in combination with certain oils and thus it can be used as an alternative natural additive in chitosan films to produce more effective antimicrobial packages. However improvement of antimicrobial effect of chitosan films could be resulted by benzaldehyde in oil extracts. In conclusion, addition of fig seed oil into food packages may extend the shelf life of a food by keeping it fresh along days.

Acknowledgements. This study was funded by The Scientific and Technological Research Council of Turkey (TUBITAK) with grantapplication number 1919B011903109 in terms of 2209-A Research Project Support Programme for Undergraduate Students. We also thank Meryem Kevser Zelka (MSc) for her motivation to work and help in the begining of our research.

\section{References}

Alamri A., El-Newehy M. H. \& Al-Deyab S. S. (2012), Biocidal polymers: synthesis and antimicrobial properties of benzaldehyde derivatives immobilized onto amineterminated polyacrylonitrile, Chemistry Central Journal, 6(1), pp. 111, DOI: 10.1186/1752153X-6-111.

Amit S. K., Uddin M. M., Rahman R., Islam S. M. R. \& Khan M. S. (2017), A review on mechanisms and commercial aspects of food preservation and processing, Agriculture \& Food Security, 6(1), pp. 51, DOI: 10.1186/s40066-017-0130-8.

Bayly C. I., Merz K. M., Ferguson D. M., Cornell W. D., Fox T., Caldwell J. W., Kollman P. A., Cieplak P., Gould I. R. \& Spellmeyer D. C. (1995), A Second Generation Force Field for the Simulation of Proteins, Nucleic Acids, and Organic Molecules, Journal of the American Chemical Society, 117(19), pp. 5179-5197, DOI: 10.1021/ja00124a002.

Chouhan S., Sharma K. \& Guleria S. (2017), Antimicrobial Activity of Some Essential Oils-Present Status and Future Perspectives, Medicines (Basel), 4(3), pp. 58, DOI: 10.3390/medicines4030058.

Debnath S., Habibur Rahman S. M., Deshmukh G., Duganath N., Pranitha C. \& Chiranjeevi, A. (2011), Antimicrobial Screening of Various Fruit Seed Extracts, Pharmacognosy Journal, 3(19), pp. 83-86, DOI: 10.5530/pj.2011.19.15.

Duman E., Şimşek M. \& Özcan M. M. (2018), Monitoring of Composition and Antimicrobial Activity of Fig (Ficus carica L.) Fruit and Seed Oil, Journal of Agroalimentary Processes and Technologies, 24(2), pp. 75-80.

Erkmen O. \& Özcan M. (2004), Antimicrobial effects of essential oils on growth of bacteria, yeasts and molds, Journal of Essential Oil Bearing Plants, 7(3), pp. 279-287, DOI: 10.1080/0972-060X.2004.10643408. 
Essien E. E., Newby J. S., Walker T. M., Setzer W. N. \& Ekundayo O. (2015), Characterization and Antimicrobial Activity of Volatile Constituents from Fresh Fruits of Alchornea cordifolia and Canthium subcordatum, Medicines, 3(1), pp. 1, DOI: 10.3390/medicines3010001.

Geng H., Yu X., Lu A., Cao H., Zhou B., Zhou L. \& Zhao Z. (2016), Extraction, Chemical Composition, and Antifungal Activity of Essential Oil of Bitter Almond, International Journal of Molecular Sciences, 17(9), pp. 1421, DOI: 10.3390/ijms17091421.

Goy R. C., De Britto D. \& Assis O. B. G. (2009), A review of the antimicrobial activity of chitosan, Polimeros, 19(3), pp. 241-247, DOI: 10.1590/S0104-14282009000300013.

Heggers J. P., Cottingham J., Gusman J., Reagor L., McCoy L. \& Carino E. (2004), The effectiveness of processed grapefruit-seed extract as an antibacterial agent: II. Mechanism of action and in vitro toxicity, The Journal of Alternative Complementary Medicine, 8(3), pp. 333-340, DOI: 10.1089/10755530260128023.

Hu Y., Du Y., Yang J., Tang Y., Li J. \& Wang X. (2007), Self-aggregation and antibacterial activity of $\mathrm{N}$-acylated chitosan, Polymer, 48(11), pp. 3098-3106, DOI: 10.1016/j.polymer.2007.03.063.

Huang T., Qian Y., Wei J. \& Zhou C. (2019), Polymeric Antimicrobial Food Packaging and Its Applications, Polymers, 11(3), pp. 560, DOI: 10.3390/polym11030560.

Inta O., Yoksan R. \& Limtrakul J. (2014), Hydrophobically modified chitosan: a biobased material for antimicrobial active film, Material Science Engineering C-Materials for Biological Applications, 42, pp. 569-577, DOI: 10.1016/j.msec.2014.05.076.

Jumaa M., Furkert F. H. \& Müller B. W. (2002), A new lipid emulsion formulation with high antimicrobial efficacy using chitosan, European Journal of Pharmaceutics and Biopharmaceutics, 53(1), pp. 115-123, DOI: 10.1016/S0939-6411(01)00191-6.

Kahve H. İ. \& Duran A. (2016), The Use Of Chitosan As A Coating Material, Journal of Academic Sciences, 5(1), pp. 167-172.

Kong F. \& Singh R. P. (2011), Chemical deterioration and physical instability of foods and beverages, In: D Kilcast \& P Subramaniam (Eds.), Food and Beverage Stability and Shelf Life, Woodhead Publishing, Cambridge, pp. 29-62.

Kong M., Chen X. G., Xing K. \& Park H. J. (2010), Antimicrobial properties of chitosan and mode of action: a state of the art review, International Journal of Food Microbiology, 144(1), pp. 51-63, DOI: 10.1016/j.ijfoodmicro.2010.09.012.

Lee H., Ahn J. H., Kwon A. R., Lee E. S., Kwak J. H. \& Min Y. H. (2014), Chemical Composition and Antimicrobial Activity of the Essential Oil of Apricot Seed, Phytotherapy Research, 28(12), pp. 1867-1872, DOI: 10.1002/ptr.5219.

Mahira S., Jain A., Khan W. \& Domb A. J. (2019), Antimicrobial Materials-An Overview, In: A J Domb K R Kunduru \& S Farah, Antimicrobial Materials for Biomedical Applications, The Royal Society of Chemistry, London, pp. 1-37.

Malhotra B., Keshwani A. \& Kharkwal H. (2015), Antimicrobial Food Packaging: Potential \& Pitfalls, Frontiers in Microbiology, 6, pp. 611, DOI: 10.3389/fmicb.2015.00611

Marpu S. B. \& Benton E. N. (2018), Shining Light on Chitosan: A Review on the Usage of Chitosan for Photonics and Nanomaterials Research, International Journal of Molecular Sciences, 19(6), pp. 1795, DOI: 10.3390/ijms19061795.

Mujić I., Bavcon-Kralj M., Jokić S., Jug T., Subarić D., Vidović S., Zivković J. \& Jarni K. (2014), Characterisation of volatiles in dried white varieties figs (Ficus carica L.), Journal of Food Science and Technology, 51(9), pp. 1837-1846, DOI: 10.1007/s13197-012-0740-x.

Odeyemi O. A., Alegbeleye O. O., Strateva M. \& Stratev D. (2020), Understanding spoilage microbial community and spoilage mechanisms in foods of animal origin, 
Comprehensive Reviews in Food Science and Food Safety, 19(2), pp. 311-331, DOI: 10.1111/1541-4337.12526.

Plimpton S. (1995), Fast parallel algorithms for short-range molecular Dynamics, Journal of Computational Physics, 117(1), pp. 1-19, DOI: 10.1006/jcph.1995.1039.

Priyadarshi R., Sauraj S., Kumar B., Deeba F., Kulshreshtha A. \& Negi Y. S. (2018), Chitosan films incorporated with Apricot (Prunus armeniaca) kernel essential oil as active food packaging material, Food Hydrocolloids, 85(22), pp. 158-166, DOI: 10.1016/j.foodhyd.2018.07.003.

Rabea E. I., Badawy M. E. T., Stevens C. V., Smagghe G. \& Steurbaut W. (2003), Chitosan as antimicrobial agent: Applications and mode of action, Biomacromolecules, 4(6), pp. 1457-1465, DOI: 10.1021/bm034130m.

Rawat S. (2015), Food Spoilage: Microorganisms and their prevention, Asian Journal of Plant Science and Research, 5(4), pp. 47-56.

Rolim F. R. L., dos Santos K. M. O., de Barcelos S. C., do Egito A. S., Ribeiro T. S., da Conceição M. L., et al. (2015), Survival of Lactobacillus rhamnosus EM1107 in simulated gastrointestinal conditions and its inhibitory effect against pathogenic bacteria in semi-hard goat cheese, LWT - Food Science and Technology, 63(2), pp. 807-813.

Russo F., Caporaso N., Paduano A. \& Sacchi R. (2017), Characterisation of volatile compounds in Cilento (Italy) figs (Ficus carica L.) cv. Dottato as affected by the drying process, International Journal of Food Properties, 20(2), pp. 1366-1376, DOI: 10.1080/10942912.2017.1344991.

Sahariah P. \& Másson M. (2017), Antimicrobial Chitosan and Chitosan Derivatives: A Review of the Structure-Activity Relationship, Biomacromolecules, 18(11), pp. 3846-3868, DOI: 10.1021/acs.biomac.7b01058.

Savic I. M., Nikolic V., Savic-Gajic I., Kundaković T., Tanojkovic T. \& Najman S. (2016), Chemical composition and biological activity of the plum seed extract, Advanced Technologies, 5(2), pp. 38-45.

Sperber W. H. (2009), Introduction to the Microbiological Spoilage of Foods and Beverages. In: W Sperber \& M Doyle (Eds.), Compendium of the Microbiological Spoilage of Foods and Beverages, Springer, New York, pp. 1-40.

Tamer T., Hassan M. A., Omer A. M., Baset W. M., Hassan M. E., El-Shafeey M. \& Eldin M. (2016), Synthesis, characterization and antimicrobial evaluation of two aromatic chitosan Schiff base derivatives, Process Biochemistry, 51(10), pp. 1721-1730, DOI: 10.1016/j.procbio.2016.08.002.

Tan Y. M., Lim S. H., Tay B. Y., Lee M. W. \& Thian E. S. (2015), Functional chitosanbased grapefruit seed extract composite films for applications in food packaging technology, Materials Research Bulletin, 69, pp. 142-146, DOI: 10.1016/j.materresbull.2014.11.041.

Thien D. T., Tuyen N. V. \& Nhi T. T. Y. (2017), Studies On Chitosan - Benzaldehyde Interaction In A Homogeneous System And Its Adsorption Behavior Of Metal Ions In Aqueous Solution, Asean Journal on Science and Technology for Development, 25(1), pp. 149, DOI: 10.29037/ajstd.238.

Verma R. S., Padalia R. C., Singh V. R., Goswami P., Chauhan A. \& Bhukya B. (2017), Natural benzaldehyde from Prunus persica (L.) Batsch, International Journal of Food Properties, 20(2), pp. 1259-1263, DOI: 10.1080/10942912.2017.1338728.

Vyviurska O., Matura F., Furdíková K. \& Špánik I. (2017), Volatile fingerprinting of the plum brandies produced from different fruit varieties, Journal of Food Science and Technology, 54(13), pp. 4284-4301, DOI: 10.1007/s13197-017-2900-5. 